\title{
Buckypaper Templating Ni-Co Hydroxide Nanosheets Used as a Sensitive Strain Sensor and Flexible Supercapacitor
}

\author{
Xin-Xue WANG ${ }^{1,2, a}$ and Tian-Shu SONG ${ }^{1, b,{ }^{*}}$ \\ ${ }^{1}$ School of Aerospace and Civil Engineering, Harbin Engineering University, Harbin, P.R.China \\ 150001 \\ ${ }^{2}$ Mudanjiang Normal University, Mudanjiang, P.R.China 157011. \\ axinxuewang_1985@126.com, bxiwang_hit@126.com \\ ${ }^{\star}$ Corresponding author
}

Keywords: Buckypaper, Ni-Co Hydroxide, Strain Sensor, Supercapacitor

\begin{abstract}
Ni-Co hydroxide nanosheets (NCHs) deposition on Buckypaper (BP) were fabricated by a facial and one-step electrodeposition method for sensitive strain sensor and high-performance supercapacitor electrodes. The binary metal hydroxides show great superiority over the related single hydroxides This NCHs/BP material exhibits a sequence of excellent properties, such as linear relationship between normalized electrical resistance $\left(\mathrm{R} / \mathrm{R}_{0}\right)$ responses to mechanical deformation, high areal capacitance of $1.5 \mathrm{~F} \mathrm{~cm}^{-2}$, high rate capability and excellent cyclic stability. The as-prepared asymmetric device shows high capacitance, low internal resistance and high flexibility (negligible capacitance changes during large deformation). These demonstrated superiority of $\mathrm{NCHs} / \mathrm{BP}$ material suggesting its promising applications as electromechanical sensors and next generation high-performance energy storage devices.
\end{abstract}

\section{Introduction}

As the today's increasing demands for multifunctional electronic devices, their power supplies are expected to become more flexible and large capacitance as well as sensitive response to environment stimuli-inputs, presenting promising applications in electrochemical sensors, biomedical devices, smart actuators and emery storage devices [1-6]. The responsive behaviors to different stimuli input, including temperature, stress, and $\mathrm{pH}$ change, to name a few, make them feasible to prepare smart materials with special functions. Moreover, among numerous energy storage systems, supercapacitors have attracted many attentions because of its fast power delivery, high rate capability and stable cyclic life compared to those of traditional supercapacitors featuring active materials on current collector substrates with whole device inevitably bulky [7-11]. Recently, film-like Buckypaper of carbon nanomaterials have been developed to bring unprecedented opportunities for the flexible electronic devices and next-generation high-performance supercapacitors since these devices become ultrathin, light, flexible and easily scalable [12-14]. Diverse carbon nanostructures with abundant resource, high conductivity, and structural stability have been widely used as supercapacitor electrodes and strain sensors [4, 5, 15-17]. The carbon nanomaterials synthesized with carbon nanotubes (CNTs) are low cost while the carbon fibers with micro-diameter have relatively low specific surface area. In comparison, another commercially available carbon nano-structure based material-buckypaper (made by CNTs), with large specific surface area, mechanical flexibility, high electrical conductivity and also cost benefit, suggests its promising in applications of strain sensors and flexible supercapacitor electrodes [18-20].

In this work, $\mathrm{Ni}-\mathrm{Co}$ hydroxide nanosheets $\left(\mathrm{NCH}_{\mathrm{S}}\right)$ on $\mathrm{BP}$ was fabricated using a facial and one-step electrodeposition method with a unique sheets-on-tubes hierarchical microstructure. The morphology and structure of the $\mathrm{NCH}_{\mathrm{S}} / \mathrm{BP}$ composite were characterized by scanning electron microscope (SEM), X-ray diffraction (XRD) and X-ray photoelectron spectroscopy (XPS), demonstrating a uniform distribution of NCHS on BP with enlarged specific surface area. Such NCHS/BP hybrid composites exhibit excellent mechanical robustness, high sensitivity to mechanical 
deformation stimuli, large specific surface areal capacitance, high rate capability and stable cyclic life in a three-electrode configuration. Such as-prepared NCHS/BP devices show great promising applications in the mechanical-stimulated sensors and next-generation energy storage systems with multi-functionality and high scalability.

\section{Materials and Method}

The NCHs/BP film was prepared by a facial one-step electrodeposition method as reported in previous work [21]. The BP was purchase from Nanolab Inc (USA), and sulfuric acid, $\mathrm{Ni}\left(\mathrm{NO}_{3}\right)_{2} \cdot 6 \mathrm{H} 2 \mathrm{O}, \mathrm{Co}\left(\mathrm{NO}_{3}\right)_{2} \cdot 6 \mathrm{H}_{2} \mathrm{O}$ and polyethylene terephthalate film were purchased from local supplier. At first, the BP was electrochemically oxidized in $\mathrm{H}_{2} \mathrm{SO}_{4}$ solution $(1 \mathrm{M})$ in an attempt to make it hydrophilic. After that, the electrodeposition was conducted using a conventional three-electrode system consisting of the as-treated BP as the working electrode, a platinum mesh as the counter electrode and a saturated calomel electrode (SCE) as the reference electrode. A constant potential of $-1.0 \mathrm{~V}$ vs. SCE was adopted to electrodeposit the NCHs upon the BP in an aqueous solution containing $0.1 \mathrm{Mol} \mathrm{Ni}\left(\mathrm{NO}_{3}\right)_{2}$ and $0.03 \mathrm{Mol} \mathrm{Co}\left(\mathrm{NO}_{3}\right)_{2}$. After electrodeposition for 2 mins, the final NCHs/BP film was obtained after rinsing several times with deionized water. For electromechanical test, the as-prepared $\mathrm{NCHs} / \mathrm{BP}$ film was firstly cut into small pieces by a size of $10 \times 20 \times 0.075 \mathrm{~mm}$, and was electrically insulated and packaged at two sides by $0.5 \mathrm{~mm}$ thick polyethylene terephthalate film after the setting up of copper tape for conductive electrode. Asymmetric supercapacitors were assembled using NCHS/BP as the positive electrode and bare BP as the negative electrode (The total mass of the positive and negative electrodes are 2.99 and $2.12 \mathrm{mg}$ $\mathrm{cm}^{-2}$, respectively). The KOH/PVA polymer gel was used as both electrolyte and separator [21]. The electrodes with the electrolyte solution coating on were placed in a fume hood at room temperature to evaporate the excess water. After the KOH/PVA became solidified, the two electrodes were tightly pressed together into one integrated unit.

The surface morphology of the NCHs/BP was investigated by field emission scanning electron microscope (SEM, ZEISS Merlin) with an operating voltage of $10 \mathrm{~kW}$. X-ray diffraction analysis was carried out by an X-ray diffractometer (Rigaku Smartlab) using $\mathrm{Cu}-\mathrm{K} \alpha$ source (1.54 $\AA$ ). X-ray photoelectron spectroscopy (XPS, PHI 5700 ESCA System) was obtained on an SSI Probe XPS spectrometer with an $\mathrm{Al}(\mathrm{K} \alpha)$ source. And polyethylene terephthalate film packaged NCHs/BP film was test the electromechanical responses using a typical two-electrode method. The electrochemical performance of electrodes was evaluated using a Gamry Echem Testing System (Gamry Instruments, Inc., USA). Electrochemical measurements were conducted in a three-electrode configuration at room temperature using $2 \mathrm{Mol} \mathrm{KOH}$ as electrolyte for hybrid electrodes. The asymmetric supercapacitor devices were electrochemically characterized in a two-electrode configuration cell.

\section{Results and Discussion}

\section{Characterizations of Microstructure and Chemical Composition}

The morphology of NCHS/BP has been characterized by SEM as shown in Fig. 1. Before electrodepositing process, numerous CNTs interconnect, forming a network (see Fig. 1a). The BP provides a light, flexible, and mechanically robust substrate for NDHS deposition. Figure S2, indicating the potential of BP applied in electrochemical fields. As shown in Fig. 1b, the surface of CNTs presents smooth morphology, and such CNTs exhibits diameters of approx. 20 30 nm. After electrodeposition of $\mathrm{NDH}_{\mathrm{S}}$ for $2 \mathrm{~min}$, the CNTs surface becomes fluffy, covered by a uniform layer of NCHs (see Fig. 1c). The high-magnification SEM image in Fig. 1d reveals the NCHs with a lateral size of several tens of nanometre. 

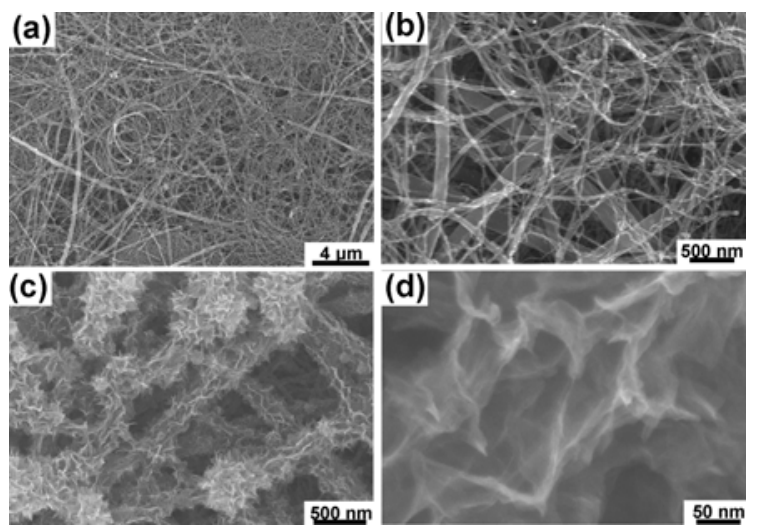

(d)

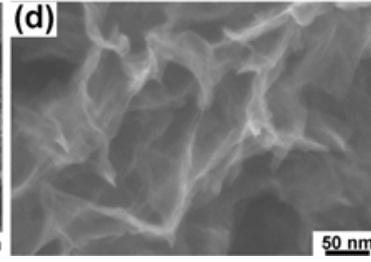

Fig. 1 Microstructural morphologies of (a) Bare BP at low magnification; (b) CNT network at high magnification; (c) Uniform distribution of NCHs on BP; (d) NCHs on BP at high magnification.

The XRD pattern of the bare BP and NCHs/BP is shown in Fig. 2a. The highly intense (002) peak at $26.4^{\circ}$ confirms the graphite structure from the BP precursor. Both diffraction peaks at $36.8^{\circ}$ and $42.8^{\circ}$ are ascribed to the XRD pattern of the $\mathrm{NiCoO}_{2}$ sample. Meanwhile, the four characteristic peaks at $34.9^{\circ}, 39.5^{\circ}, 53.1^{\circ}$ and $60.9^{\circ}$ is attributed from the crystal structures of comblainite $\left(\mathrm{Ni} 6.10 \mathrm{Co} 2.90(\mathrm{OH})_{18.27}\left(\mathrm{CO}_{3}\right)_{1.315} \cdot 6.7 \mathrm{H}_{2} \mathrm{O}\right)$ according to the JCPDS no. 33-0429. And the peaks located at $31.1^{\circ}$ and $44.6^{\circ}$ correspond to the (220) and (400) plane reflections of the $\mathrm{Co}_{2} \mathrm{NiO}_{4}$ sample. From the XRD patterns, the formation of the $\mathrm{NCHs}$ during the electrodeposit process can be proved. The chemical composition of NCHs/BP film was studied by XPS analysis, as shown in Fig. 2b-d. The peaks located at 284.90, 530.91, 780.91 and $855.91 \mathrm{eV}$ are corresponding to $\mathrm{C}_{1 \mathrm{~s}}, \mathrm{O}_{1 \mathrm{~s}}, \mathrm{Co}_{2 \mathrm{p}}$ and $\mathrm{Ni}_{2 \mathrm{p}}$, and the three fitting peaks of $\mathrm{C}_{1 \mathrm{~s}}$ spectrum of Fig.2c corresponding to non-functionalized graphic structures (C-C, $286.4 \mathrm{eV}$ ), C-O functional groups (286.4 and $288.7 \mathrm{eV})$, respectively. In the $\mathrm{O}_{1 \mathrm{~s}}$ spectrum of Fig. $2 \mathrm{~d}$, the peak at $530.5 \mathrm{eV}$ is a typical peak of metal-oxygen bonds, while the peaks at 531.6 and 533.2 $\mathrm{eV}$ are related to $\mathrm{C}-\mathrm{O}$ functional groups.
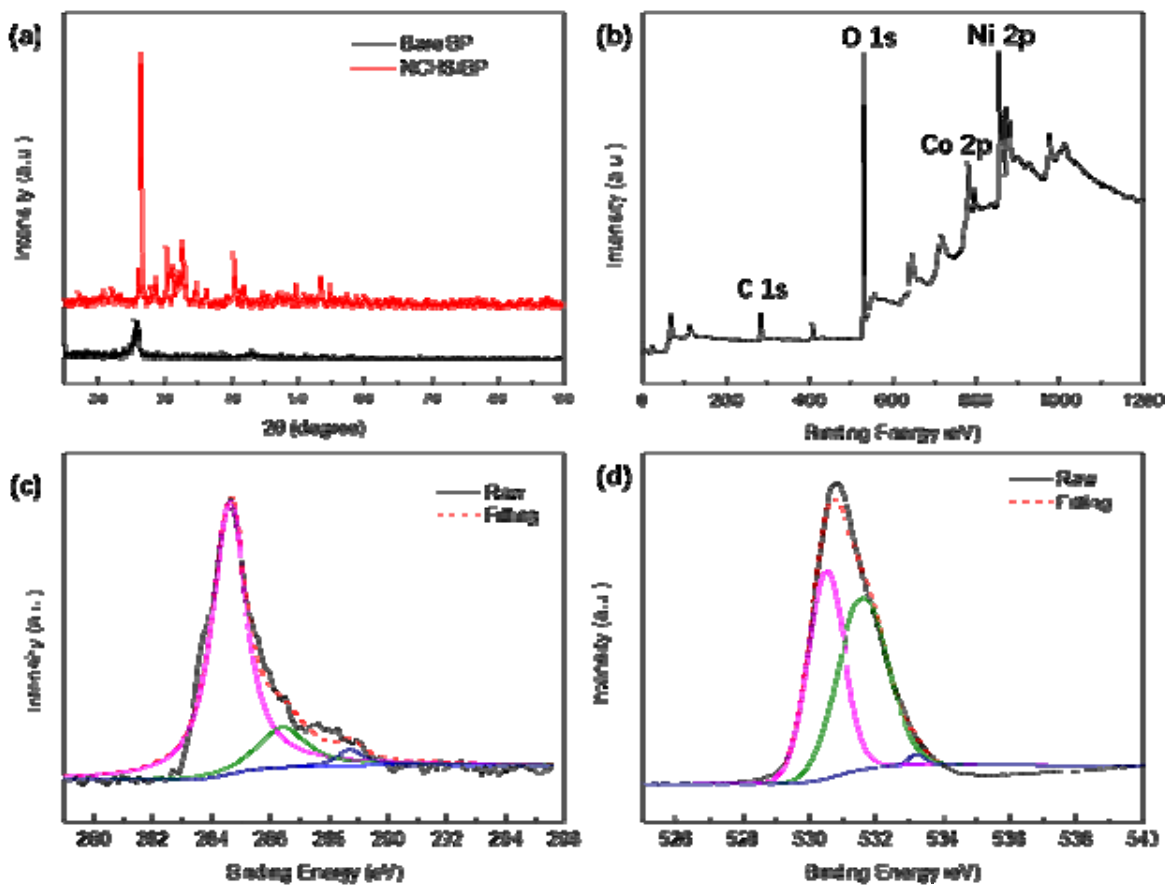

Fig.2 (a) XRD patterns of bare BP and NCHs/BP; (b) Wide-scan of XPS spectrum of NCHs/BP; (c) $\mathrm{C}_{1 \mathrm{~s}} ;$ (d) $\mathrm{O}_{1 \mathrm{~s}}$. 


\section{Mechanical-stimulated Sensing Performance}

Fig.3a-b illustrates the electro-mechanical responses of the NCHs/BP film under different stimuli types (stretching, bending and torsion). As shown in the inner plot, the two cupper electrodes of test sample were connected to a digital to monitor and record the data of the electrical resistance under specific stimuli input. A linearly dependent behavior between normalized electrical resistance $\left(\mathrm{R} / \mathrm{R}_{0}\right)$ and tensile strain is presented in Fig. 3a, exhibiting a tensile strain responsive property of the $\mathrm{NCHs} / \mathrm{BP}$ film. What's more, in a smaller tensile strain of $2.0 \%$, over $80 \%$ of electrical resistance variation $(\Delta R / R)$ produced, suggesting a highly sensitive performance of this $\mathrm{NCHs} / \mathrm{BP}$ film. The stretching-releasing cycle test under excitation of square wave by different frequencies $(0.1 \mathrm{~Hz}$ and $0.5 \mathrm{~Hz}$, respectively) is adopted to evaluate the cyclic stability of the NCHs/BP film, which presents decent recoverability and stability in 20 cycles as shown in Fig. 3b. Furthermore, the capabilities of NCHs/BP film respond to mechanical bending and torsion have also been evaluated by bending and torsion angle. Fig. $3 \mathrm{c}-\mathrm{d}$ show the variations of the $\mathrm{R} / \mathrm{R}_{0}$ values towards bending and torsion angle, similar to the above strain-related normalized electrical resistance. The $\mathrm{R} / \mathrm{R}_{0}$ values are basically proportional to the bending or torsion angle. The large electrical resistance variation amplitudes of $36.0 \%\left(0-180^{\circ}\right)$ and $70.6 \%\left(0-90^{\circ}\right)$, bending and torsion test, respectively, can be employed for precise monitoring for mechanical deformation. As a consequence, the obvious electrical resistance variation to variant mechanical stimuli, makes NCHs/BP film an excellent candidate for mechanical sensor applications.
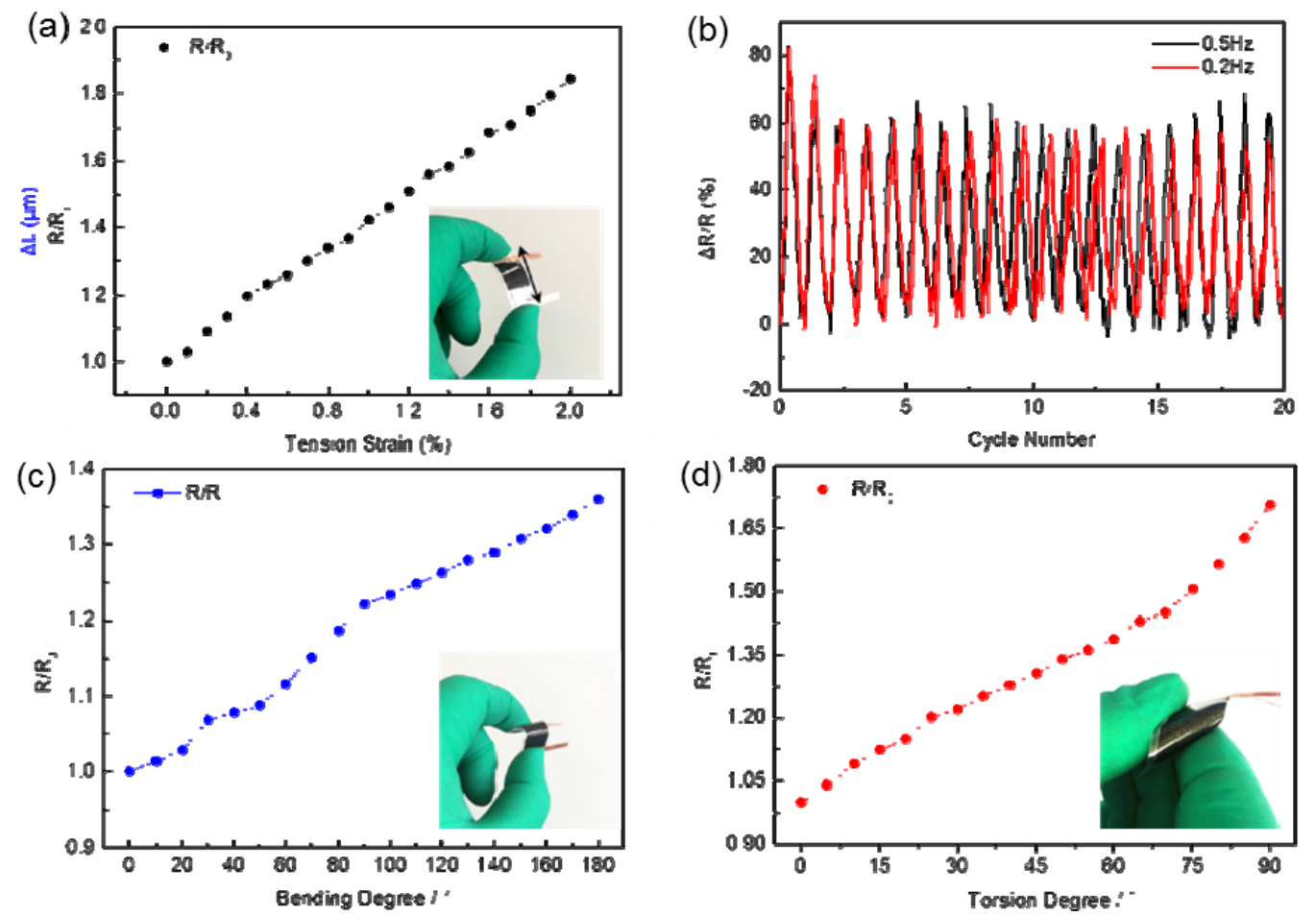

Fig. 3 The electro-mechanical properties of $\mathrm{NCHs} / \mathrm{BP}$. (a) R/Ro vs. stretching stain; (b) The stretching-releasing cycle test under square wave excitation by different frequencies; (c-d) Normalized electrical resistance $\left(\mathrm{R} / \mathrm{R}_{0}\right)$ vs. different mechanical stimulis of bending and torsion, respectively.

\section{Asymmetric Supercapacitor Device Performance}

Fig. 4 characterizes electrochemical performance of the NCHs/BP hybrid electrodes. Fig. $4 \mathrm{a}$ shows typical CV profiles at scan rates from 2 to $40 \mathrm{mV} \mathrm{s}^{-1}$ with a potential window from -0.2 to $0.5 \mathrm{~V}$ vs. $\mathrm{SCE}$ in $2 \mathrm{Mol} \mathrm{KOH}$ aqueous electrolyte. Two clear redox peaks appear in the CV curves 
corresponding to the redox reactions between NCHs and the alkaline electrolyte. Fig. $4 \mathrm{~b}$ provides the galvanostatic charge/discharge profiles of $\mathrm{NCHs} / \mathrm{BP}$ electrodes at different current densities ranging from 2 to $12 \mathrm{~mA} \mathrm{~cm}^{-2}$. These constant-current charge/discharge curves display a relatively symmetric shape, indicating high coulombic efficiency and the reversible nature of the redox reactions. There are two voltage plateaus in the charge/discharge curves, which are consistent with the voltages of redox peaks in the $\mathrm{CV}$ curves. Accordingly, the capacitance of NCHs/BP electrodes is $1.5 \mathrm{~F} \mathrm{~cm}^{-2}$ calculated from the charge/discharge curves. The capacitance retention has been plotted as a function of different current densities as shown in Fig. 4c. The capacitance decreases gradually with the increase of current densities, and the NCHS/BP electrode exhibits $62 \%$ capacitance retention at a high current density of $50 \mathrm{~mA} \mathrm{~cm}$. The cyclic life is also an important factor affecting the performance of electrodes in practical applications. The NCHs/BP electrodes were tested under 3,000 galvanostatic charge/discharge cycles at a current density of $15 \mathrm{~mA} \mathrm{~cm}^{-2}$ from 0 to $0.4 \mathrm{~V}$ as shown in Fig. 4d. The hybrid electrodes exhibit a slight elevating in capacitance during the first 200 cycles and then undergo a mild degeneration until the $3,000^{\text {th }}$ cycle with final capacitance retention of approx. $88 \%$ compared with the $1^{\text {st }}$ cycle. The increase of capacitance in the initial cycle was observed in many metal oxide/hydroxide electrodes, which is hypothetically explained by the gradually ameliorated electrolyte penetrating/wetting the electrodes and structural activation of the active materials [22]. Moreover, the hybrid electrode exhibits high coulombic efficiencies ( $>99 \%)$ over the 3,000 cycles, indicating high charge transfer efficiencies over long-term cycling serves.

Moreover, The flexibility is a crucial property for a supercapacitor in practical applications. The high flexibility of as-prepared asymmetric supercapacitor has been demonstrated in Fig. 4e-f. The CV curves of the device under different deformation states (see Fig. 4e) have been plotted in Fig. 4b. Obviously, the CV curves show slight changes under different bending states, even under seriously twisted and folded deformation, indicating the high flexibility and excellent stability of the as-prepared asymmetric supercapacitor.
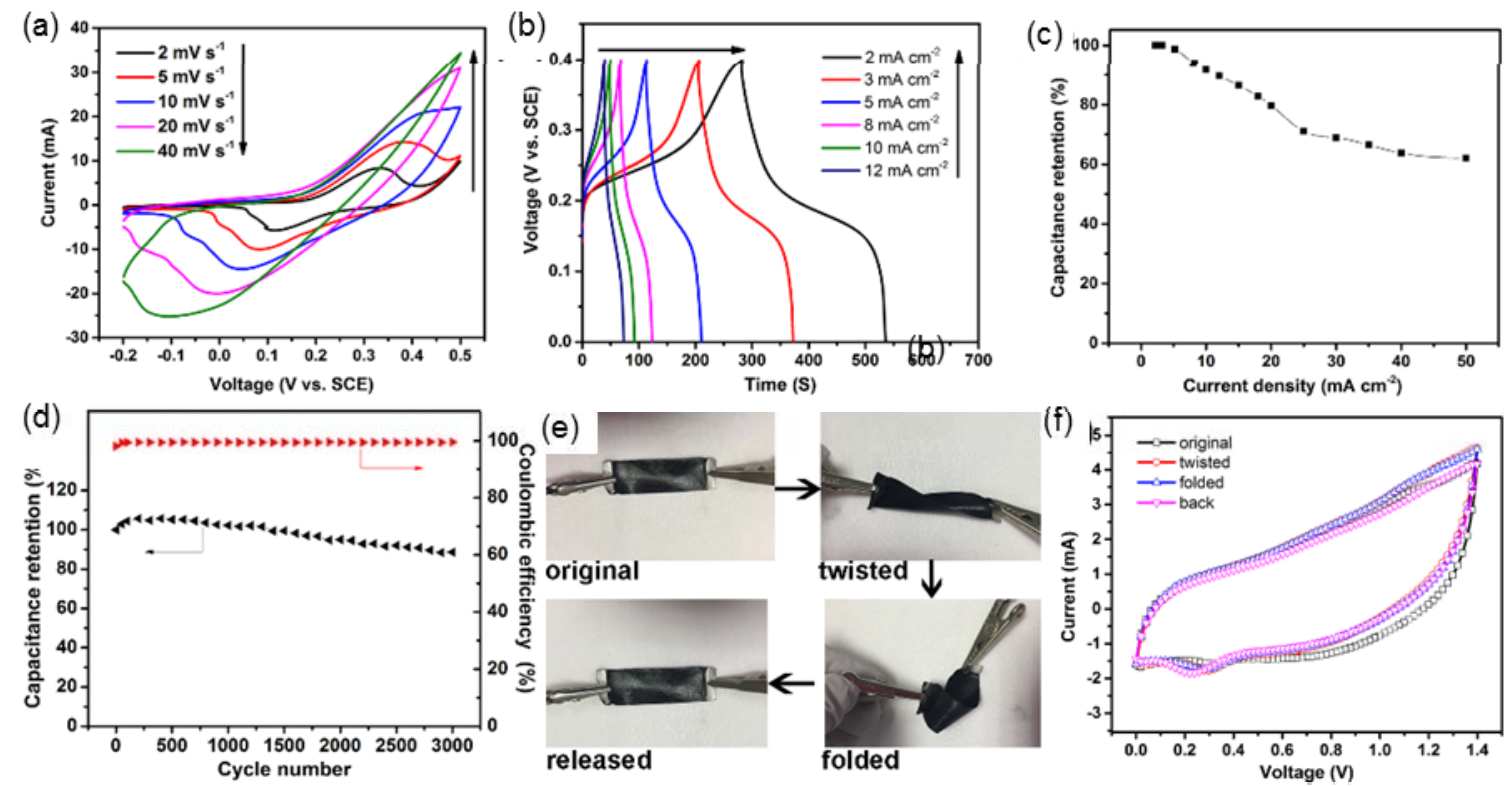

Fig. 4 Electrochemical and flexibility characterization of NCHS/BP electrodes. (a) CV curves at scan rates from 2 to $40 \mathrm{mV} \mathrm{s}-1$; (d) Galvanostatic charge/discharge curves at low current densities from 2 to $12 \mathrm{~mA} \mathrm{~cm}-2$ in the voltage range between 0 and $0.4 \mathrm{~V}$ vs. SCE; (c) The capacitance retention as a

function of current densities; (d) Cyclic stability test at a current density of $25 \mathrm{~mA} \mathrm{~cm}-2$ and corresponding coulombic efficiencies over 3,000 cycles from 0 to $0.4 \mathrm{~V}$ vs. SCE; (e) The snapshots for the devices under different deformation states; (f) CV curves measured at a scan rate of $20 \mathrm{mV} \mathrm{s}^{-1}$ under the corresponding deformation state in (e). 


\section{Summary}

In this article, we highlight a Ni-Co hydroxide nanosheets (NCHs) deposited on Buckypaper (BP), which was fabricated by a facial and one-step electrodeposition method for sensitive strain sensor and high-performance supercapacitor electrodes. Through the mechanical deformation induced stimuli-responsive investigation and in situ electrochemical test, this NCHs/BP material exhibits a sequence of excellent properties, such as linear relationship between normalized electrical resistance $\left(\mathrm{R} / \mathrm{R}_{0}\right)$ responses to mechanical deformation, high areal capacitance of $1.5 \mathrm{~F} \mathrm{~cm}^{-2}$, high rate capability and excellent cyclic stability. The as-prepared asymmetric device shows high capacitance, low internal resistance and high flexibility (negligible capacitance changes during large deformation). These demonstrated superiority of NCHs/BP material suggesting its promising applications as electromechanical sensors and next generation high-performance energy storage devices.

\section{Acknowledgement}

The authors gratefully acknowledge all help and discussion involved in this work.

\section{References}

[1] L. Qiu, D. Liu, Y. Wang, C. Cheng, K. Zhou, J. Ding, V.T. Truong, D. Li, Mechanically robust, electrically conductive and stimuli-responsive binary network hydrogels enabled by superelastic graphene aerogels, Adv. Mater. 26 (2014) 3333-3337.

[2] D. Selvakumar, A. Alsalme, A. Alghamdi, R. Jayavel, Reduced graphene oxide paper as bimorphic electrical actuators, Mater. Lett. 191 (2017) 182-185.

[3] J. Yun, D.H. Lee, J.S. Im, H. Il Kim, Improvement in transdermal drug delivery performance by graphite oxide/temperature-responsive hydrogel composites with micro heater, Mater. Sci. Eng. C. 32 (2012) 1564-1570.

[4] Q. Zhang, X. Xu, H. Li, G. Xiong, H. Hu, T.S. Fisher, Mechanically robust honeycomb graphene aerogel multifunctional polymer composites, Carbon 93 (2015) 659-670

[5] Q. Zhang, X. Xu, D. Lin, W. Chen, G. Xiong, Y. Yu, T.S. Fisher, H. Li, Hyperbolically Patterned 3D Graphene Metamaterial with Negative Poisson's Ratio and Superelasticity, Adv. Mater. 28 (2016) 2229-2237

[6] P. Simon, Y. Gogotsi, Materials for electrochemical capacitors, Nat. Mater. 7 (2008) 845-854.

[7] M. Winter, R. J. Brodd, What are batteries, fuel cells, and supercapacitors? Chem. Rev. 104 (2004) 4245-4270.

[8] Q. Wu, Y. Xu, Z. Yao, A. Liu, G. Shi, Supercapacitors based on flexible graphene/polyaniline nanofiber composite films, ACS Nano 4 (2010) 1963-1970.

[9] K. Wang, P. Zhao, X. Zhou, H. Wu, Z. Wei, Flexible supercapacitors based on cloth-supported electrodes of conducting polymer nanowire array/SWCNT composites, J. Mater. Chem. 21 (2011) 16373-16378.

[10] S. Ye, J. Feng, P. Wu, Deposition of three-dimensional graphene aerogel on nickel foam as a binder-free supercapacitor electrode, ACS Appl. Mater. Inter. 5 (2013) 7122-7129.

[11]K. Kalinathan, D. P. DesRoches, X. Liu, P. G. Pickup, Anthraquinone modified carbon fabric supercapacitors with improved energy and power densities, J. Power Sources 181 (2008) 182-185.

[12]G. Zheng, L. Hu, H. Wu, X. Xie, Y. Cui, Paper supercapacitors by a solvent-free drawing method, Energy Environ. Sci. 4 (2011) 3368-3373. 
[13] Y. J. Kang, H. Chung, C. H. Han, W. Kim, All-solid-state flexible supercapacitors based on papers coated with carbon nanotubes and ionic-liquid-based gel electrolytes, Nanotechnology 23 (2012) 065401.

[14]Z. Weng, Y. Su, D. W. Wang, F. Li, J. Du, H. M. Cheng, Graphene-cellulose paper flexible supercapacitors, Adv. Energy Mater. 1 (2011) 917-922.

[15]A. Pandolfo, A. Hollenkamp, Carbon properties and their role in supercapacitors, J. Power Sources 157 (2006) 11-27.

[16] P. He, L. Liu, W. Song, G. Xiong, T. S. Fisher, T. Chen, Large-scale synthesis and activation of polygonal carbon nanofibers with thin ribbon-like structures for supercapacitor electrodes, RSC Advances 5 (2015) 31837-31844.

[17] S. Vivekchand, C. S. Rout, K. Subrahmanyam, A. Govindaraj, C. Rao, Graphene-based electrochemical supercapacitors, Journal of Chemical Sciences 120 (2008) 9-13.

[18] S. He, J. Wei, F. Guo, R. Xu, C. Li, X. Cui, H. Zhu, K. Wang, D. Wu, A large area, flexible polyaniline/buckypaper composite with a core-shell structure for efficient supercapacitors, J. Mater. Chem. A 2 (2014) 5898-5902.

[19]H. Chen, J. Di, Y. Jin, M. Chen, J. Tian, Q. Li, Active carbon wrapped carbon nanotube buckypaper for the electrode of electrochemical supercapacitors, J. Power Sources 237 (2013) 325-331.

[20] J. Che, P. Chen, M. B. Chan-Park, High-strength carbon nanotube buckypaper composites as applied to free-standing electrodes for supercapacitors, J. Mater. Chem. A 1 (2013) 4057-4066.

[21] X. Wang, T. Song, Buckypaper templating Ni-Co hydroxide nanosheets as free-standing electrodes for ultrathin and flexible supercapacitors, New J. Chem. 40 (2016) 8006-8011.

[22] S. K. Meher, G. R. Rao, Ultralayered $\mathrm{Co}_{3} \mathrm{O}_{4}$ for high-performance supercapacitor applications, J. Phys. Chem. C, 115 (2011) 15646-15654. 